\title{
Growth of high crystalline quality semi-insulating GaN layers for high electron mobility transistor applications
}

Hongbo Yu, Deniz Caliskan, and Ekmel Ozbay

Citation: Journal of Applied Physics 100, 033501 (2006); doi: 10.1063/1.2221520

View online: http://dx.doi.org/10.1063/1.2221520

View Table of Contents: http://aip.scitation.org/toc/jap/100/3

Published by the American Institute of Physics

\section{Articles you may be interested in}

Growth of Fe doped semi-insulating GaN by metalorganic chemical vapor deposition

Applied Physics Letters 81, 439 (2002); 10.1063/1.1490396

Polarization effects, surface states, and the source of electrons in AIGaN/GaN heterostructure field effect transistors

Applied Physics Letters 77, 250 (2000); 10.1063/1.126940

Semi-insulating C-doped GaN and high-mobility AIGaN/GaN heterostructures grown by ammonia molecular beam epitaxy

Applied Physics Letters 75, 953 (1999); 10.1063/1.124252

On the physical operation and optimization of the p-GaN gate in normally-off GaN HEMT devices

Applied Physics Letters 110, 123502 (2017); 10.1063/1.4978690

Electrical and optical properties of Fe-doped semi-insulating GaN templates

Applied Physics Letters 83, 3314 (2003); 10.1063/1.1621458

Role of carbon in GaN

Journal of Applied Physics 92, 6553 (2002); 10.1063/1.1518794

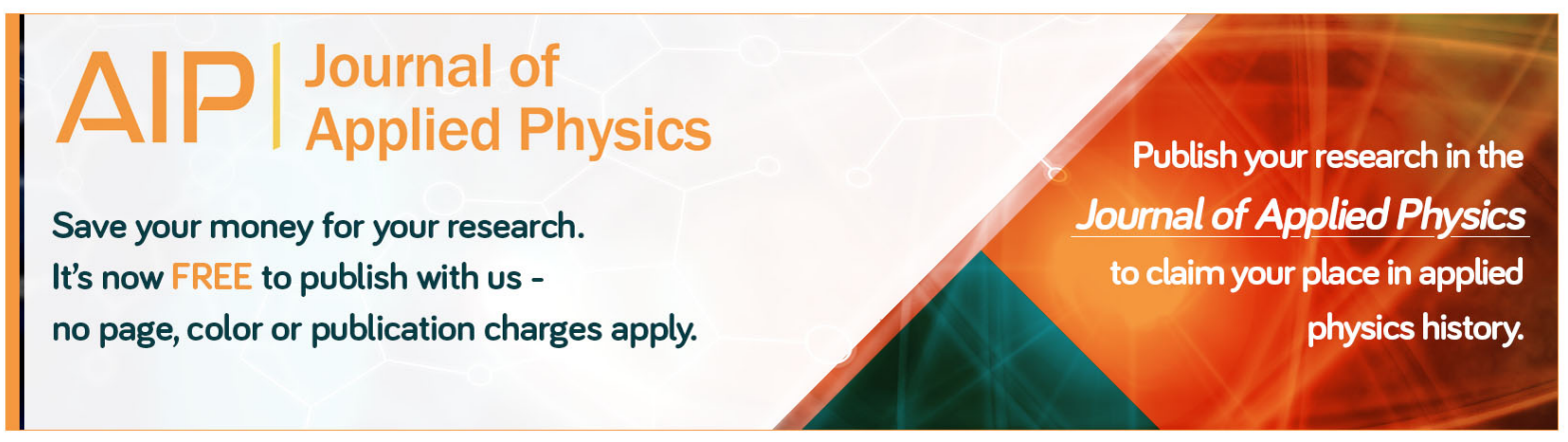




\title{
Growth of high crystalline quality semi-insulating GaN layers for high electron mobility transistor applications
}

\author{
Hongbo $\mathrm{Yu}^{\mathrm{a})}$ and Deniz Caliskan \\ Nanotechnology Research Center, Bilkent University, Bilkent, 06800 Ankara, Turkey \\ Ekmel Ozbay \\ Nanotechnology Research Center, Bilkent University, Bilkent, 06800 Ankara, Turkey; Department \\ of Physics, Bilkent University, Bilkent, 06800 Ankara, Turkey; and Department of Electrical \\ and Electronics Engineering, Bilkent University, Bilkent, 06800 Ankara, Turkey
}

(Received 15 December 2005; accepted 6 June 2006; published online 1 August 2006)

\begin{abstract}
Semi-insulating character (sheet resistivity of $3.26 \times 10^{11} \Omega / \mathrm{sq}$ ) of thick GaN layers was developed for $\mathrm{AlGaN} / \mathrm{GaN}$ high electron mobility transistor (HEMT) applications on an AlN buffer layer. Electrical and structural properties were characterized by a dark current-voltage transmission line model, x-ray diffraction, and atomic force microscope measurements. The experimental results showed that compared to semi-insulating GaN grown on low temperature GaN nucleation, the crystal quality as well as surface morphology were remarkably improved. It was ascribed to the utilization of a high quality insulating AlN buffer layer and the GaN initial coalescence growth mode. Moreover, the significant increase of electron mobility in a HEMT structure suggests that this is a very promising method to obtain high performance AlGaN/GaN HEMT structures on sapphire substrates. (C) 2006 American Institute of Physics. [DOI: 10.1063/1.2221520]
\end{abstract}

\section{INTRODUCTION}

III-nitride based high electron mobility transistor (HEMT) has exhibited great potential for high-frequency and high-power applications. One key technique in achieving this kind of electrical device is the deposition of thick semiinsulating (SI) GaN layers, in order to ensure proper drainsource current saturation, complete channel pinchoff, and low loss at high frequencies. ${ }^{1}$ However, it is well known that the usual undoped GaN epilayers exhibit an $n$-type background. This effect is particularly evident in $\mathrm{GaN}$ that is grown on sapphire, which is the most commonly used substrate for the growth of III-nitride due to its low price, stability at high temperatures, and its mature growth technology. The origination of the $n$-type background is still unclear at present. It is reported that the SI GaN can be achieved on sapphire substrate by means of $\mathrm{C}, \mathrm{Fe}$, or $\mathrm{Zn}$ doping and the control of the initial growth procedures. ${ }^{2-8}$ However, the high-resistance characteristic is achieved due to the compensating acceptor levels that are introduced through deep-level acceptor, edge-type threading dislocations (TDs), or other defects. The existence of additional deep traps is responsible for the leakage current and trapping effect, that may adversely affect the device reliability in AlGaN/GaN HEMT. Therefore, it is quite important to develop a technique that can obtain a thick SI GaN layer without degrading the crystalline quality.

In this paper, we report the integration of the $\mathrm{GaN}$ epigrowth with a high quality AlN buffer layer. $2 \mu \mathrm{m}$ thick SI $\mathrm{GaN}$ was grown on AlN template/sapphire substrate by the metal-organic chemical vapor deposition (MOCVD) technique. Based on electrical and structural studies, SI electrical properties (sheet resistivity of $3.26 \times 10^{11} \Omega / \mathrm{sq}$ ) as well as

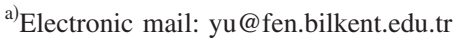

better crystalline quality of GaN were achieved, when compared to the normal GaN grown on sapphire by the two-step method.

\section{EXPERIMENT}

The III-nitride epilayers were grown on $c$-plane sapphire $\left(\mathrm{Al}_{2} \mathrm{O}_{3}\right)$ substrates in a low pressure MOCVD reactor. Standard trimethylgallium (TMGa), trimethylaluminum (TMAl), and ammonia were used as $\mathrm{Ga}, \mathrm{Al}$, and $\mathrm{N}$ precursors, respectively. Three GaN samples were grown using different procedures: sample A was grown on $0.5 \mu \mathrm{m}$ high temperature (HT) AlN buffer, while samples B and C were grown on low temperature (LT) GaN nucleation layers (NLs), in which the nucleation and anneal processes were different. Prior to the epilayer growth, the sapphire substrates were cleaned in $\mathrm{H}_{2}$ ambient at $1100{ }^{\circ} \mathrm{C}$ for $10 \mathrm{~min}$. For sample A, a $15 \mathrm{~nm}$ thick LT AlN NL was grown at $840{ }^{\circ} \mathrm{C}$. The temperature was then ramped to $1150{ }^{\circ} \mathrm{C}$ and the sample was annealed at this temperature for $2.5 \mathrm{~min}$. This was followed by the growth of a $0.5 \mu \mathrm{m}$ HT AlN buffer layer. This structure was used as a template for growth of a $2.0 \mu \mathrm{m}$ thick undoped $\mathrm{GaN}$ layer. For comparison, a high-resistance GaN layer was also grown on LT GaN NL (sample B). This high-resistance GaN was achieved by calibrating the thicknesses of LT-NL and the HT annealing process carefully. ${ }^{1,5}$ Sample $\mathrm{C}$ was grown as a reference sample by using the standard two-step growth conditions. The growth parameters of the HT GaN main layer were kept constant for all of the samples.

High resolution $\mathrm{x}$-ray diffraction (XRD) and atomic force microscope (AFM) measurements were carried out to characterize the crystalline quality of the epitaxial layers. The sheet resistivities of the high-resistance GaN layers were measured by an HP 4142B semiconductor parameter analyzer using the transmission line method (TLM). The TLM 


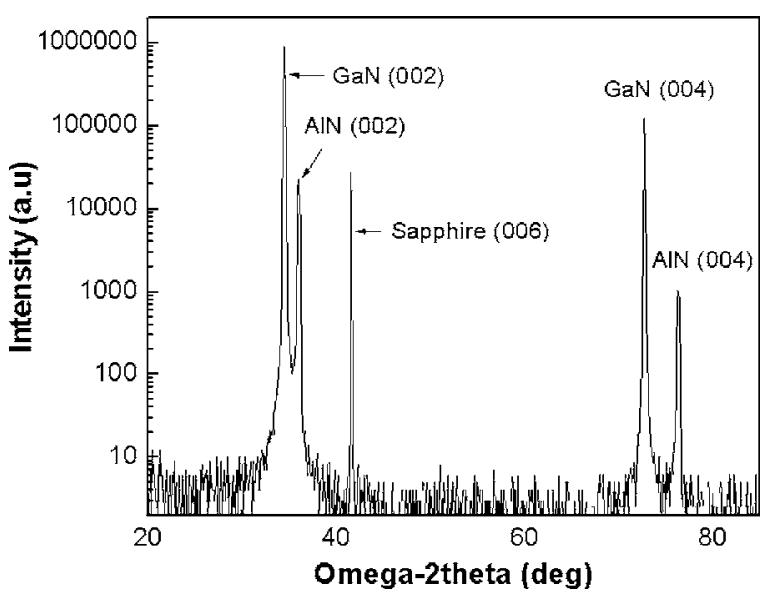

FIG. 1. XRD $\omega / 2 \theta$ scan of sample A.

patterns consisted of a set of $200 \times 100 \mu \mathrm{m}^{2}$ pads with space in the 5-50 $\mu \mathrm{m}$ range. A thin $(25 \mathrm{~nm}) \mathrm{Al}_{0.3} \mathrm{Ga}_{0.7} \mathrm{~N}$ layer was grown on the SI GaN samples. The presence of twodimensional electron gas at the $\mathrm{AlGaN} / \mathrm{GaN}$ interface ensures an Ohmic contact, as proposed in Ref. 2. The Ohmic contacts were prepared by the deposition of $\mathrm{Ti} / \mathrm{Al} / \mathrm{Ni} / \mathrm{Au}$ $(100 \AA / 400 \AA / 100 \AA / 400 \AA)$. After this, the AlGaN layer between the contacts was etched off with reactive ion etching. In situ interferometry was used to monitor the growth process and to measure the growth rate for each sample.

\section{RESULTS AND DISCUSSIONS}

A symmetric $\omega / 2 \theta \mathrm{x}$-ray diffractometer was used to evaluate the structure of the as-grown GaN layer on an AlN buffer from $20^{\circ}$ to $85^{\circ}$ diffraction angles. As can be seen in Fig. 1, sharp sapphire peaks can be used as a reference signal. Strong GaN (002) and AlN (002) peaks are displayed approximately at the $34.5^{\circ}$ and $36^{\circ}$, respectively, as well as high indices, such as GaN (004) and AlN (004). No other plane reflections were detected. After optimization, the AlN layer exhibited narrow full width at half maximum (FWHM) for both rocking and $\omega / 2 \theta$ scan peaks: 323 arc sec for rocking scan and 298 arc sec for $\omega / 2 \theta$ scan, respectively. ${ }^{10}$

Figure 2 shows the $I-V$ characteristics of $\mathrm{GaN}$ grown on AlN buffer obtained from a set of TLM pads. After rapid thermal annealing at $750{ }^{\circ} \mathrm{C}$ for $1 \mathrm{~min}$, the linearity of $I-V$ curves was obtained for all the TLM pads. At a bias of $10 \mathrm{~V}$, the dark remains about $2 \times 10^{9}$ A between two TLM pads separated by a $5 \mu \mathrm{m}$ gap. The $I-V$ characteristics demonstrate that a GaN layer grown on AlN buffer/sapphire substrate exhibits a semi-insulating character with calculated

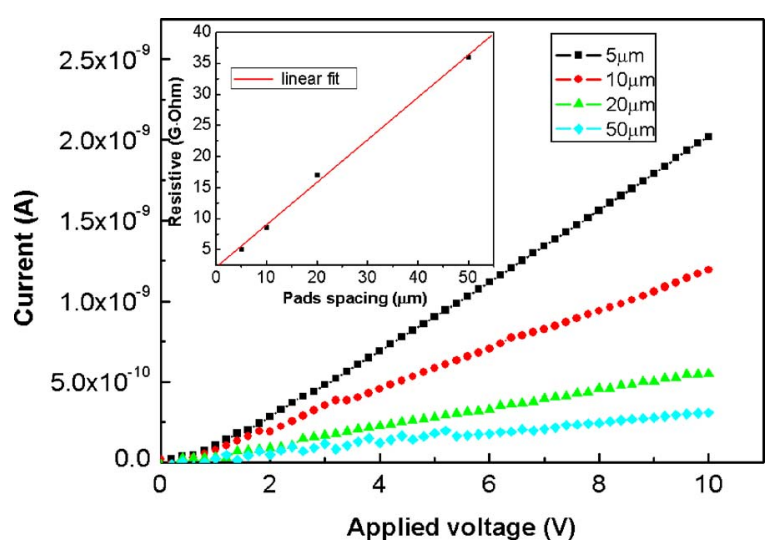

FIG. 2. (Color online) Dark $I-V$ characteristics of sample A obtained from a set of TLM pads.

sheet resistivity of $3.26 \times 10^{11} \Omega /$ sq, which was one order of magnitude higher than the previous reported results in the literature. ${ }^{1}$

Table I summarizes the sheet resistivity and FWHM of XRD peaks from different samples. The rocking scans were measured around the (002) and (102) reflections of the GaN layers. Samples A and B show SI electrical properties, with sheet resistivities of $3.26 \times 10^{11}$ and $2.7 \times 10^{11} \Omega /$ sq, respectively. The normally undoped $\mathrm{GaN}$ layer (sample $\mathrm{C}$ ) shows an $n$-type background with sheet resistivity of 9.77 $\times 10^{3} \Omega /$ sq (electron concentration of $1.6 \times 10^{16} \mathrm{~cm}^{-3}$ ). We suspected that the $n$-type background of $\mathrm{GaN}$ grown on sapphire is not due to the impurity incorporation during HT growth, because the growth parameters of the HT-GaN main layer were kept constant for the SI GaN and normal GaN. It is most likely that the $n$-type background of GaN epilayers is originated from the sapphire substrate or the LT-GaN nucleation growth process. During the initial growth stage, oxygen can move into the GaN layer by thermal etching and/or diffusion, which results in a highly conducting channel near the sapphire interface. ${ }^{11,12}$ So there are two main ways to obtain SI electrical character of GaN grown on sapphire substrate: (i) intentional introduction of acceptor-type level in the band gap to compensate the $n$-type background. However, this method may also result in high density of defects in the epilayers; (ii) insertion of insulating layer to block off the effect from sapphire substrate which acts as the oxygen source. The electrical character and crystal quality of subsequent $\mathrm{GaN}$ epilayer is influenced by the characteristic of insulating material definitively.

XRD symmetric (002) incorporated with asymmetric (102) scan is a reliable technique to characterize the crystal quality of GaN films. The defects within the structure of sample B cause significant broadening in both the symmetric

TABLE I. Sheet resistivities and FWHM of XRD peaks from different samples.

\begin{tabular}{cccccc}
\hline \hline Sample & $\begin{array}{c}\text { Nucleation } \\
\text { Layer }\end{array}$ & Buffer layer & $\begin{array}{c}\text { Sheet resistivity } \\
(\Omega / \mathrm{sq})\end{array}$ & $\begin{array}{c}\text { FWHM of rocking } \\
\text { scan }(0002)(\operatorname{arcsec})\end{array}$ & $\begin{array}{c}\text { FWHM of rocking } \\
\text { scan }(10-12)(\operatorname{arcsec})\end{array}$ \\
\hline A & LT-AlN & HT-AlN & $3.26 \times 10^{11}$ & 234 & 280 \\
B & LT-GaN & No & $2.7 \times 10^{11}$ & 360 & 1026 \\
C & LT-GaN & No & $9.77 \times 10^{3}$ & 256 & 312 \\
\hline \hline
\end{tabular}



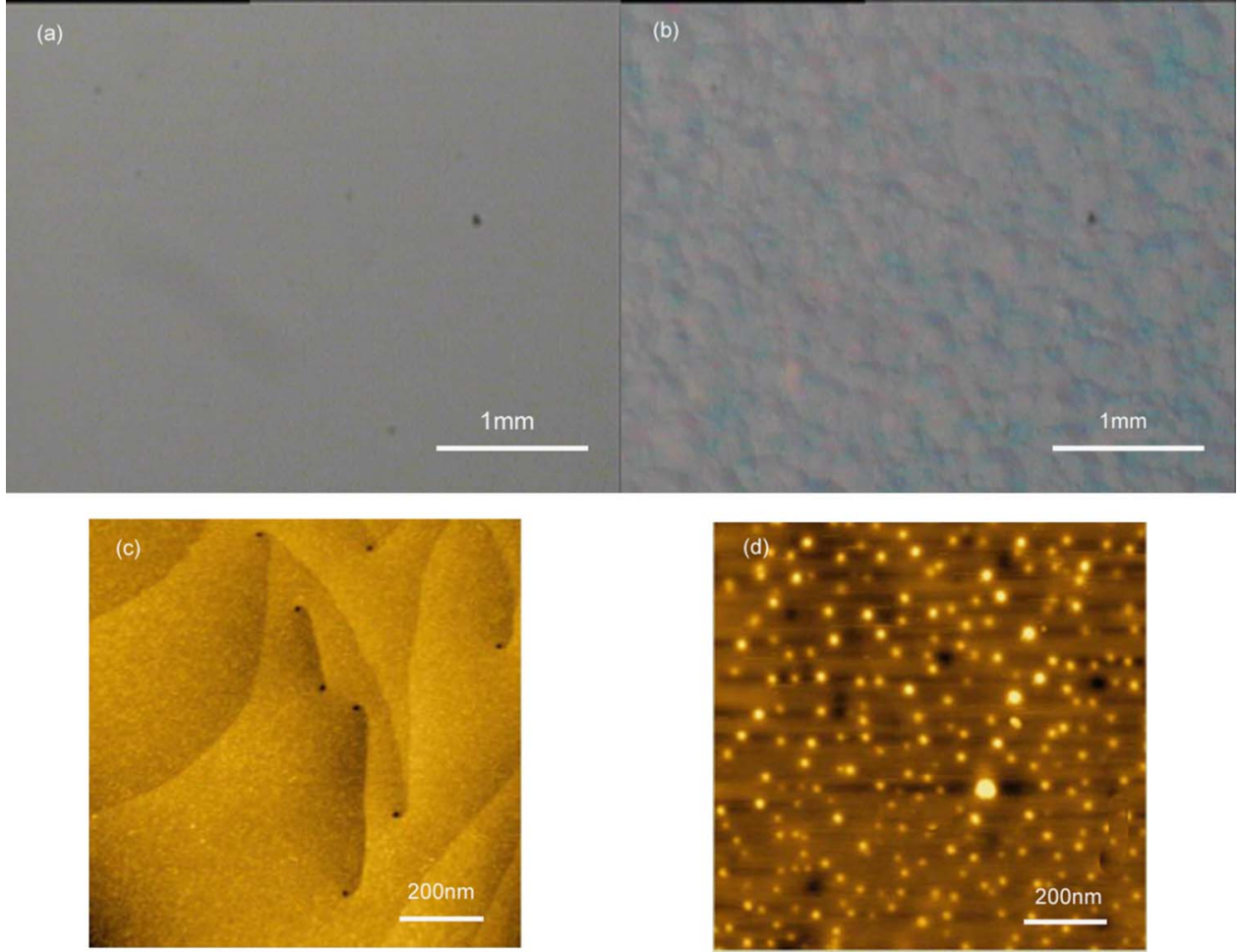

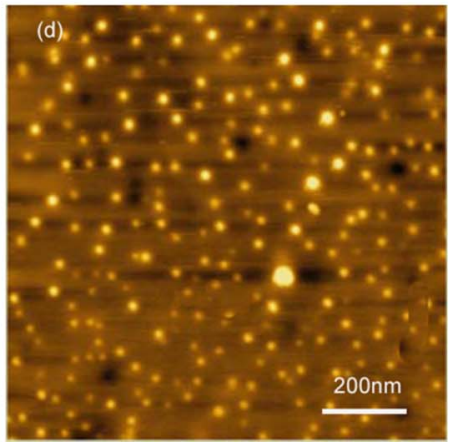

FIG. 3. (Color online) Nomarski optical microscope and $1 \times 1 \mu \mathrm{m}^{2}$ area AFM images of as-grown GaN surface for [(a) and (c)] sample A and [(b) and (d)] sample B
(002) and asymmetric (102) rocking curves, which have fitted widths of 360 and 1026 arc sec, respectively. It is noteworthy that the measured width of the (102) rocking curve from sample B is strikingly larger than that of the (002) rocking curve. The dramatic broadening of the asymmetric diffractions compared to the symmetric diffractions is indicative of a defective structure with a large pure edge TD content, since the (002) peak is only broadened by screw or mixed TD while the (102) peak is broadened by all of the TDs. ${ }^{13}$ For sample B, it is very likely that the high-density edge-type TDs compensate the background of $\mathrm{GaN}$, leading to the SI electrical character. The (002) and (102) rocking curves have fitted widths of 234 and 280 arc sec for sample A, and 256 and 312 arc sec for sample C, respectively. Compared to that of sample $\mathrm{B}$, sample A has remarkably narrower peak widths for both (002) and (102) reflection planes indicating a significant improvement of crystalline quality. The narrow (102) peak width of SI sample A means that factors other than edge-type TDs are responsible for the SI electrical character. It is noted that both the (002) and (102) peak widths of sample A are narrower than that of sample $\mathrm{C}$, which was grown using a normal two-step method. We ascribe the crystal quality improvement to the $\mathrm{GaN}$ layer grown on high quality AlN buffer layer not on LT-NL directly.

Figure 3 is the surface image of an as-grown GaN epilayer taken by a Nomarski optical microscopy in order to compare the optical macroscopic morphology between samples A and B. Compared to the mirrorlike surface of sample A [Fig. 3(a)], the surface image of sample B shows an obvious bumpy surface morphology [Fig. 3(b)], which appears as craterlike defects. In order to further study the surface morphology of samples A and B, an AFM measurement was carried out in a $1 \times 1 \mu \mathrm{m}^{2}$ area of the as-grown
GaN layers. Sample A features a smooth surface morphology delineated by steps, terraces, and dark spots [Fig. 3(c)] with the root mean square (rms) of $0.25 \mathrm{~nm}$. In contrast, sample B [Fig. 3(d)] is dominated by pits and a high density (3 $\left.\times 10^{10} / \mathrm{cm}^{2}\right)$ of raised islandlike structures of various sizes (up to $60 \AA \mathrm{high}$ ) with the rms of $0.36 \mathrm{~nm}$. So any trace of underlying steps and terraces was washed out on the AFM map. The image of sample A shows a surface morphology with lots of terraces, which have a height of $0.3 \mathrm{~nm}$ corresponding to one monolayer of (002) GaN. Most of the terraces are pinned by small dark spots with a density of about $8 \times 10^{8} / \mathrm{cm}^{2}$. Step terminations on a single crystal surface must correspond to the intersection of a pure screw or mixed screw-edge threading dislocation with the free surface. The surface terraces suggest that a typical two-dimensional (2D) step-flow growth mode basically dominates the GaN growth of sample A, which is entirely different from that of sample B. A HT-AlN buffer and LT-GaN nucleation high-resistance $\mathrm{GaN}$ templates were used to grow $\mathrm{Al}_{0.3} \mathrm{Ga}_{0.7} \mathrm{~N} / \mathrm{AlN} / \mathrm{GaN}$ HEMT structures with $25 \mathrm{~nm} \mathrm{AlGaN}$ and $2 \mathrm{~nm}$ AlN barriers. Hall-effect measurements were performed on both samples at room temperature. The mobilities of the HT-AlN buffer and LT-GaN nucleation samples were 1700 and $1260 \mathrm{~cm}^{2} / \mathrm{V} \mathrm{s}$, respectively (sheet charge was 1.3 $\times 10^{13} \mathrm{~cm}^{-2}$ for both samples). As can be seen, the improved surface quality of the HT-AIN sample leads to evidently higher values of the mobility.

In situ reflectance can be used to relate the growth mechanisms to the sheet resistance and crystal quality. Figure 4 shows the reflectance traces of samples B and A during the epigrowth. As shown in Fig. 4, the LT-NL growth of sample $\mathrm{B}$ is from point $A$ to point $B$, and then the reactor temperature is ramped to HT for annealing. Growth of the main HT-GaN layer begins at point C. By controlling the 

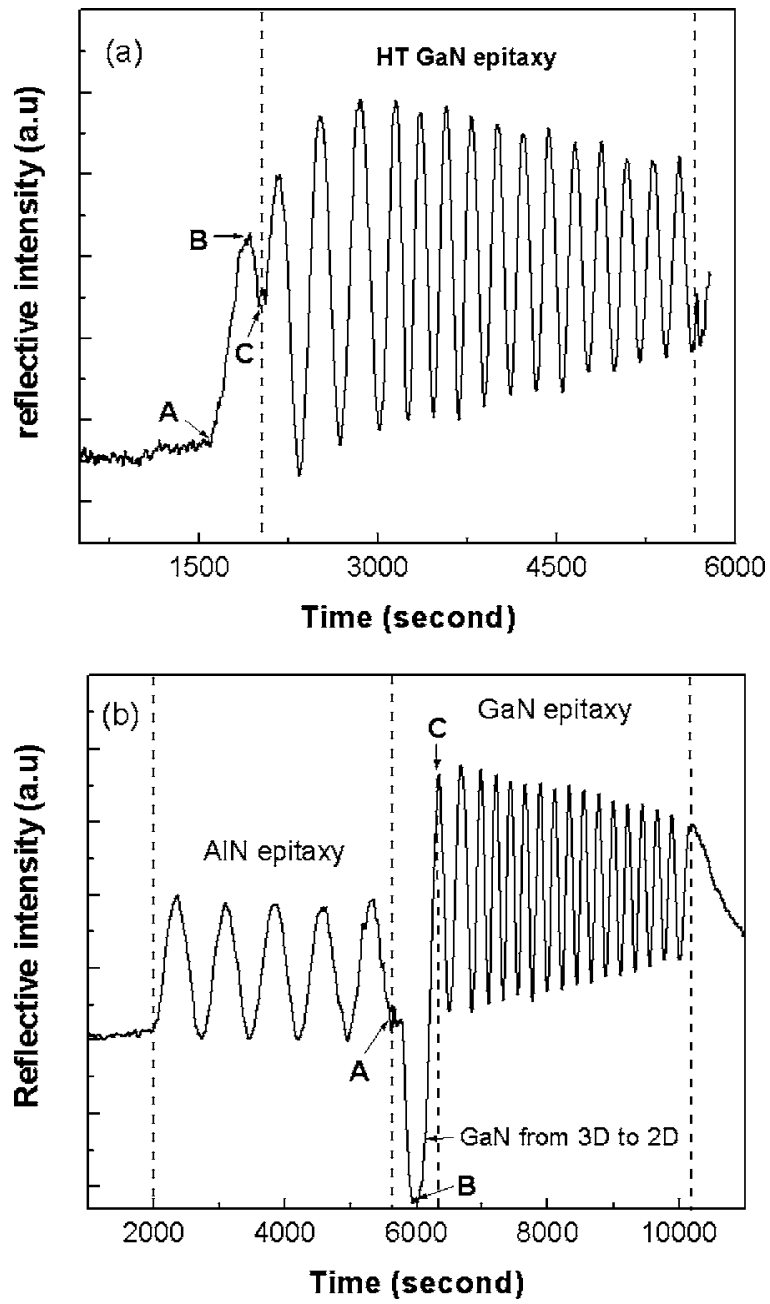

FIG. 4. Reflectivity monitored during semi-insulating GaN epitaxy on (a) LT-GaN nucleation and (b) AlN buffer. The 3D-2D transition is clearly visible in GaN grown on AlN buffer.

thickness of NL, annealing temperature, and annealing time, the $2 \mathrm{D}$ growth mode begins almost immediately after the onset of HT growth. The edge-type TDs cannot be bent by these processes, which collide with the rules of achieving high crystal quality GaN with a smooth surface. As shown in Fig. 4(b), the growth of the main $\mathrm{GaN}$ is from point $A$, and the reflectance is decreased during the initial stage (from points A to B) of HT GaN growth due to the threedimensional (3D) islandlike growth. It is ascribed to the $2.4 \%$ lattice mismatch between GaN and AlN. As the islands begin to coalesce growth (quasi-2D growth), the reflectance will begin to increase (from points $B$ to $C$ ). It is reported that the longer coalesce time (from 3D-2D growth) enables more of the edge-type TDs to annihilate each other with $90^{\circ}$ bending. ${ }^{14}$ Compared to the growth process of sample B, the initial 3D-2D growth mode of sample A can decrease the density of TDs and improve crystal quality, which is consistent with the material characterization results.

\section{SUMMARY}

In summary, we have demonstrated the MOCVD growth of thick high-resistance $\mathrm{GaN}$ layers on sapphire substrate by inserting an AlN buffer layer. The SI character (sheet resistivity of $3.26 \times 10^{11} \Omega / \mathrm{sq}$ ) of $\mathrm{GaN}$ can be achieved with high crystal quality and step-flow surface morphology. It was ascribed to the utilization of a high quality insulating AlN buffer layer and the $\mathrm{GaN}$ initial coalescence growth mode. The HEMT structure was grown based on this kind of SI GaN/AlN buffer. Hall measurement reveals that this is a very promising method to obtain high performance $\mathrm{AlGaN} / \mathrm{GaN}$ HEMT on sapphire substrate.

\section{ACKNOWLEDGMENTS}

This work was supported by EU-DALHM, EU NOEMETAMORPHOSE, EU NOE-PHOREMOST, TUBITAKNANOTR, and TUBITAK under Project Nos. 104E090 and 105E066. One of the authors (E.O.) acknowledges partial support from Turkish Academy of Sciences.

${ }^{1}$ S. M. Hubbard, G. Zhao, D. Pavlidis, W. Sutton, and E. Cho, J. Cryst. Growth 284, 297 (2005).

${ }^{2}$ S. Heikman, S. Keller, S. P. DenBaars, and U. K. Mishra, Appl. Phys. Lett. 81, 439 (2002).

${ }^{3}$ J. B. Webb, H. Tang, S. Rolfe, and J. A. Bardwell, Appl. Phys. Lett. 75, 953 (1999).

${ }^{4}$ A. Y. Polyakov, N. B. Smirnov, A. V. Govorkov, and S. J. Pearton, Appl. Phys. Lett. 83, 3314 (2003).

${ }^{5}$ Z. Bougrioua, I. Moerman, L. Nistor, B. Van Daele, E. Monroy, T. Palacios, F. Calle, and M. Leroux, Phys. Status Solidi A 195, 93 (2003).

${ }^{6}$ J.-H. Lee, M.-B. Lee, S.-H. Hahm, Y.-H. Lee, J.-H. Lee, Y.-H. Bae, and H. K. Cho, MRS Internet J. Nitride Semicond. Res. 8, 5 (2003).

${ }^{7}$ N. I. Kuznetsov, A. E. Nikolaev, I. P. Nikitina, and V. A. Dmitriev, Appl. Phys. Lett. 75, 3138 (1997).

${ }^{8}$ A. Y. Polyakov, A. V. Govorkov, N. B. Smirnov, A. E. Nikolaev, I. P. Nikitina, and V. A. Dmitriev, Solid-State Electron. 45, 249 (2001).

${ }^{9}$ D. S. Green, S. R. Gibb, B. Hosse, R. Vetury, D. E. Grider, and J. A. Smart, J. Cryst. Growth 272, 285 (2004).

${ }^{10}$ H. B. Yu, E. Ulker, and E. Ozbay, J. Cryst. Growth (accepted).

${ }^{11}$ D. C. Look and R. J. Molnar, Appl. Phys. Lett. 70, 3377 (1997).

${ }^{12}$ M. G. Cheong et al., Appl. Phys. Lett. 77, 2557 (2000).

${ }^{13}$ B. Heying, X. H. Wu, S. Keller, Y. Li, D. Kapolnek, B. P. Keller, S. P. DenBaars, and J. S. Speck, Appl. Phys. Lett. 68, 643 (1996).

${ }^{14}$ J. Chen, J. Cryst. Growth 254, 348 (2003). 